DESY 01-xxx

hep-ph/01xxxxx

\title{
The Process $\gamma_{L}^{*}+q \rightarrow(q \bar{q} g)+q:$ Real Corrections to the Virtual Photon Impact Factor
}

\author{
J. Bartels* , S. Gieseke* ${ }^{* \dagger}$ A. Kyrieleis ${ }^{\dagger}$ \\ II. Institut für Theoretische Physik, Universität Hamburg \\ Luruper Chaussee 149, 22761 Hamburg, Germany
}

\begin{abstract}
We calculate, for the longitudinally polarized virtual photon, the cross section of the process $\gamma^{*}+q \rightarrow(q \bar{q} g)+q$ at high energies with a large rapidity gap between the fragmentation system $q \bar{q} g$ and the other quark. This process provides the real corrections of the virtual photon impact factor in the nextto leading order. Evidence is given for the appearance of a new $q \bar{q} g$ Fockcomponent of the photon state.
\end{abstract}

\section{INTRODUCTION}

After the completion of the NLO virtual corrections [1], the next step in our program of computing the virtual photon impact factor in next-to-leading order is the calculation of the emission of a real gluon, as sketched in Fig. 1. We will compute these corrections from the high energy limit of the process $\gamma^{*}+q \rightarrow(q \bar{q} g)+q$ (Fig. 2) at leading order in $\alpha_{s}$. We are interested in the region of phase space, were the $q \bar{q}$-pair and the $g$ are emitted in the fragmentation region of the virtual photon with a large rapidity gap relative to the outgoing lower quark. We impose no further restrictions on the mass of the $(q \bar{q} g)$-state, and therefore our results will also include the production of the gluon in the central region: this corresponds to the leading-order BFKL calculation, where the gluon is emitted with a large rapidity gap relative to both the lower quark and to the upper $q \bar{q}$-pair. For the calculation of the photon impact factor, this piece has to be subtracted from our results.

A study of the process $\gamma^{*}+q \rightarrow(q \bar{q} g)+q$ includes the subprocess $\gamma^{*}+g \rightarrow q \bar{q} g$ with an off-shell incoming gluon. Together with the NLO corrections to the production process $\gamma^{*}+g \rightarrow q \bar{q}$ which has been calculated in [1], it provides the complete NLO corrections to the photon-gluon fusion process with off-shell incoming bosons. This generalizes the usual on-shell calculation of the collinear factorization scheme to the $k_{T}$-factorization scheme [2]. During the last years evidence has been collected that, for certain final states in the small- $x$

\footnotetext{
*supported by the EU TMR-Network 'QCD and the Deep Structure of Elementary Particles', contract number FMRX-CT98-0194 (DG 12-MIHT).

†supported by the Graduiertenkolleg 'Zukünftige Entwicklungen der Teilchenphysik'.
} 
region, the use of this scheme provides a better description of HERA data than the traditional collinear factorization scheme: for the modeling of final states it is therefore important to have the off-shell photon-gluon fusion not only in LO but also in NLO.

(a)

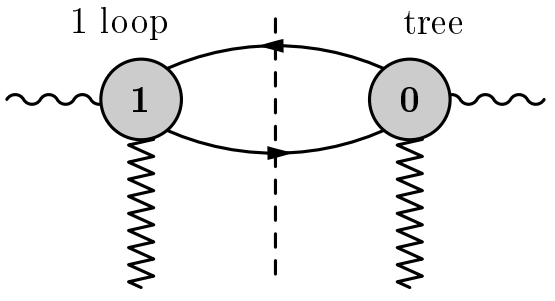

(b)

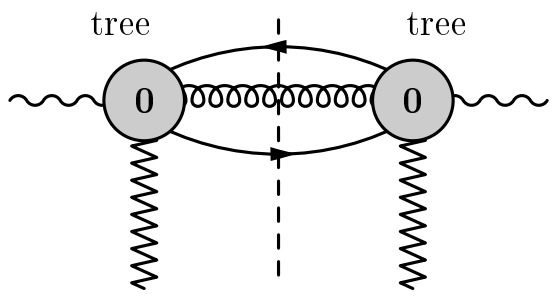

FIG. 1. Different contributions to the $\gamma^{*}$ impact factor at NLO.

Finally, it is well-known that the leading order photon impact factor can be interpreted in terms of the photon wave function and a color dipole cross section [3, 4]. In recent years this color dipole picture has proven to be extremely useful for analyzing HERA data in the small- $x$ region, in particular the low- $Q^{2}$ region of the $F_{2}$ structure function and the diffractive final states [5 77. The validity of the color dipole picture (or, maybe, its generalization to higher multipole terms) beyond leading order therefore presents an issue which has to be investigated. In NLO, the validity of the photon wave function picture is one of the results that should come out of the NLO photon impact factor calculation: in the interpretation of our results we will therefore pay particular attention to this aspect.

\section{KINEMATICS AND CALCULATIONAL TECHNIQUES}

The notations of the scattering process are depicted in Fig 2. The incoming momenta $q=q^{\prime}-x p$ and $p$ are used to construct the Sudakov basis $q^{\prime}, p$ (with $q^{\prime 2}=p^{2}=0$ ) for the other momenta, and $s=2 p \cdot q^{\prime}$ denotes the center of mass energy of the incoming particles. The virtuality of the photon is $q^{2}=-Q^{2}$, and as usual, $x=Q^{2} / s$. The momentum transfer is denoted by $r$ (with $r^{2}=t$ ), the outgoing gluon carries momentum $\ell$ and the momenta of the quark and antiquark are $k+r$ and $q-k-\ell$, respectively. We express these momenta in terms of their Sudakov variables,

$$
\begin{aligned}
& k=\alpha q^{\prime}+\beta p+k_{\perp}, \\
& \ell=\alpha_{\ell} q^{\prime}+\beta_{\ell} p+\ell_{\perp}, \\
& r=\alpha_{r} q^{\prime}+\beta_{r} p+r_{\perp},
\end{aligned}
$$

where the Euclidean notation is used for the two dimensional transverse momenta, e.g. $\boldsymbol{k}^{2}=-k_{\perp}^{2}$. We are interested in the limit of large energy $s: s$ has to be much larger than $Q^{2}, \boldsymbol{k}^{2}, \boldsymbol{\ell}^{2}$, and $\boldsymbol{r}^{2}$, and the $\alpha$ variables of the $q \bar{q} g$-system are of the order unity. The regions of integration are

$$
\begin{aligned}
\mathcal{O}(t / s) & <\alpha<1 \\
\frac{|\ell|}{\sqrt{s}}<\alpha_{\ell} & <1-\alpha .
\end{aligned}
$$




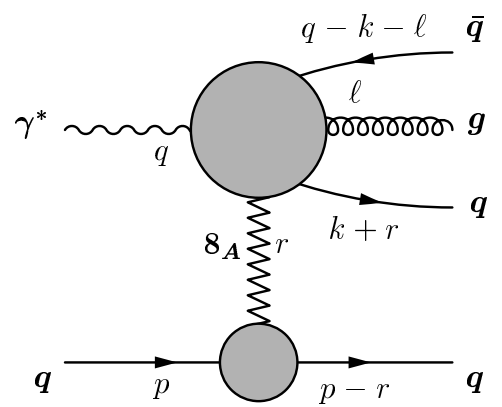

FIG. 2. Kinematics of the process $\gamma^{*}+q \rightarrow q \bar{q} g+q$.

For the produced gluon we require a large rapidity gap between the gluon and the lower quark. In other words, we include both the fragmentation region of the virtual photon

$$
\alpha_{\ell 0}<\alpha_{\ell}<1-\alpha
$$

(where $\alpha_{\ell}$ denotes a small but energy independent cutoff parameter to be specified later), and the 'upper' part of the central region

$$
\frac{|\ell|}{\sqrt{s}}<\alpha_{\ell}<\alpha_{\ell 0}
$$

There is a remaining part of the phase space which we do not consider: the 'lower' half of the central region:

$$
\frac{\ell^{2}}{s}<\alpha_{\ell}<\frac{|\ell|}{\sqrt{s}},
$$

and the fragmentation region of the lower quark. The central region belongs to the LO BFKL calculation, whereas the fragmentation region of the lower quark is part of the quark impact factor [8]. The definition of the 'upper' and 'lower' parts of the central region is somewhat arbitrary. We have chosen the 'symmetric' separation: $\alpha_{\ell}=\frac{|\ell|}{\sqrt{s}}$ corresponds to the symmetric point $M_{u}^{2}=(q+r)^{2}=M_{d}^{2}=(p+\ell-r)^{2}=\ell^{2} s$.

From the on-shell conditions of the outgoing quarks and gluons we obtain $\alpha_{r}=t / s$ and

$$
\begin{aligned}
& \beta_{\ell} s=\frac{\boldsymbol{\ell}^{2}}{\alpha_{\ell}}, \\
& \beta s=-\frac{(\boldsymbol{k}+\boldsymbol{\ell})^{2}}{1-\alpha-\alpha_{\ell}}-\frac{\boldsymbol{\ell}^{2}}{\alpha_{\ell}}-Q^{2}, \\
& \beta_{r} s=\frac{(\boldsymbol{k}+\boldsymbol{r})^{2}}{\alpha}+\frac{(\boldsymbol{k}+\boldsymbol{\ell})^{2}}{1-\alpha-\alpha_{\ell}}+\frac{\boldsymbol{\ell}^{2}}{\alpha_{\ell}}+Q^{2} .
\end{aligned}
$$

The Feynman diagrams are listed in Fig. 3. The graphs in the second line (denoted by $\left.B_{1}, \ldots, B_{5}\right)$ are obtained from the diagrams of the first line $\left(A_{1}, \ldots, A_{5}\right)$ by interchanging the quark and antiquark, i.e. by transforming $k+r \rightarrow q-k-\ell$. In terms of the Sudakov components this transformation reads: 

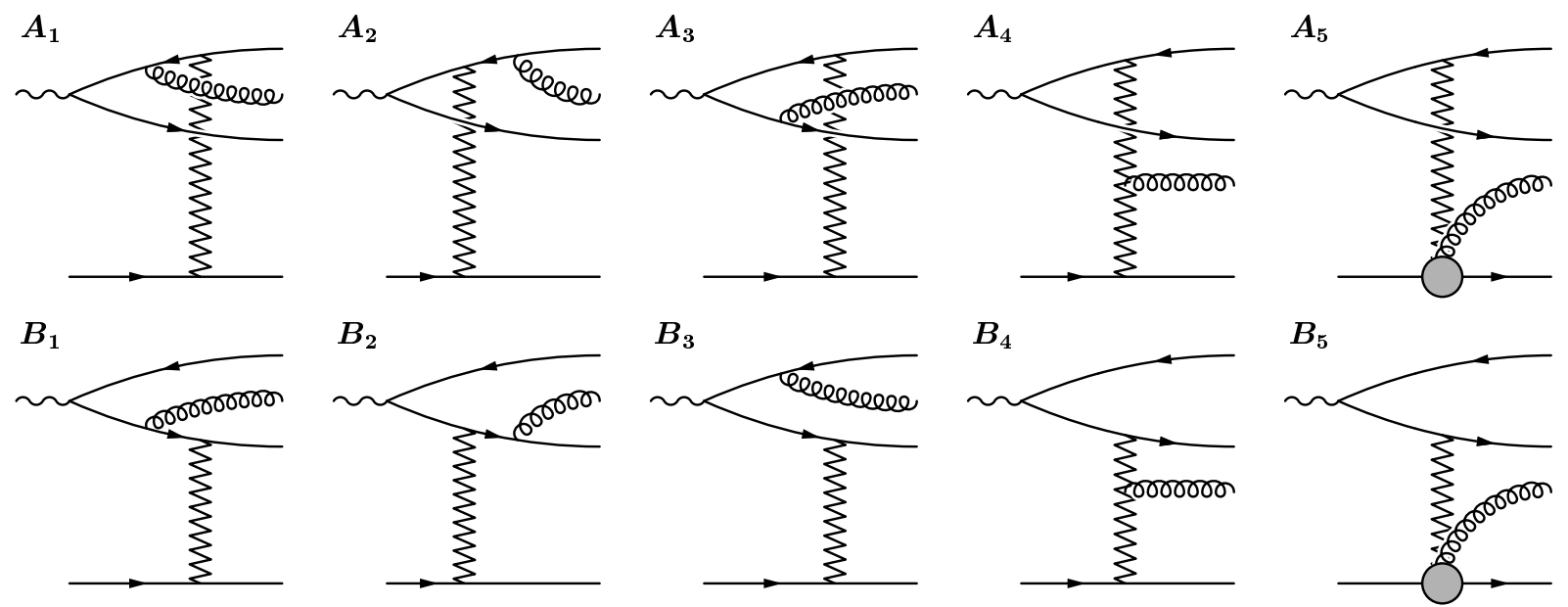

FIG. 3. Feynman diagrams for the process $\gamma^{*}+q \rightarrow q \bar{q} g+q$.

$$
\begin{aligned}
& \alpha \rightarrow 1-\alpha-\alpha_{\ell} \\
& \boldsymbol{k} \rightarrow-(\boldsymbol{k}+\boldsymbol{r}+\boldsymbol{\ell}) .
\end{aligned}
$$

In correspondence with this transformation we introduce the shorthand notation:

$$
\begin{aligned}
& \alpha_{1} \equiv \alpha, \\
& \bar{\alpha}_{1} \equiv\left(1-\alpha-\alpha_{\ell}\right), \\
& \alpha_{2} \equiv(1-\alpha), \\
& \bar{\alpha}_{2} \equiv\left(\alpha+\alpha_{\ell}\right) .
\end{aligned}
$$

where the bars stand for the transformation (111), (12). The denominators, that will occur during the calculation, have the form:

$$
\begin{aligned}
D_{1} & =(k+\ell+r-q)^{2} \\
& =-\bar{\alpha}_{1} Q^{2}-(\boldsymbol{k}+\boldsymbol{\ell}+\boldsymbol{r})^{2}-\frac{\bar{\alpha}_{1}}{\alpha_{1}}(\boldsymbol{k}+\boldsymbol{r})^{2}-\frac{\bar{\alpha}_{1}}{\alpha_{\ell}} \boldsymbol{\ell}^{2} \\
D_{2} & =(k+r-q)^{2} \\
& =-\alpha_{2} Q^{2}-\frac{1}{\alpha_{1}}(\boldsymbol{k}+\boldsymbol{r})^{2} \\
D_{3} & =(k+\ell+r)^{2} \\
& =-(\boldsymbol{k}+\boldsymbol{\ell}+\boldsymbol{r})^{2}+\frac{\bar{\alpha}_{2}}{\alpha_{1}}(\boldsymbol{k}+\boldsymbol{r})^{2}+\frac{\bar{\alpha}_{2}}{\alpha_{\ell}} \ell^{2} \\
& =\frac{1}{\alpha_{\ell} \alpha_{1}}\left(-\alpha_{\ell}(\boldsymbol{k}+\boldsymbol{r})+\alpha_{1} \ell\right)^{2} \\
D_{4} & =(k-q)^{2} \\
& =-\boldsymbol{k}^{2}+\frac{\alpha_{2}}{\bar{\alpha}_{1}}(\boldsymbol{k}+\boldsymbol{\ell})^{2}+\frac{\alpha_{2}}{\alpha_{\ell}} \ell^{2}
\end{aligned}
$$




$$
\begin{aligned}
& =\frac{1}{\alpha_{\ell} \bar{\alpha}_{1}}\left(\alpha_{\ell}(\boldsymbol{k}+\ell)+\bar{\alpha}_{1} \ell\right)^{2} \\
D_{5} & =(\ell-r)^{2} \\
& =-\alpha_{\ell} Q^{2}-\frac{\alpha_{\ell}}{\alpha_{1}}(\boldsymbol{k}+\boldsymbol{r})^{2}-\frac{\alpha_{\ell}}{\bar{\alpha}_{1}}(\boldsymbol{k}+\boldsymbol{\ell})^{2}-(\boldsymbol{\ell}-\boldsymbol{r})^{2} \\
D_{6} & =k^{2} \\
& =-\alpha_{1} Q^{2}-\boldsymbol{k}^{2}-\frac{\alpha_{1}}{\bar{\alpha}_{1}}(\boldsymbol{k}+\boldsymbol{\ell})^{2}-\frac{\alpha_{1}}{\alpha_{\ell}} \boldsymbol{\ell}^{2} \\
D_{7} & =(k+\ell)^{2} \\
& =-\bar{\alpha}_{2} Q^{2}-\frac{1}{\bar{\alpha}_{1}}(\boldsymbol{k}+\boldsymbol{\ell})^{2} .
\end{aligned}
$$

Under the transformation (11)-(12) the denominators $D_{1}$ to $D_{7}$ obey the following relations:

$$
\begin{aligned}
& D_{1}=\bar{D}_{6}, \\
& D_{2}=\bar{D}_{7}, \\
& D_{3}=\bar{D}_{4}, \\
& D_{5}=\bar{D}_{5} .
\end{aligned}
$$

Rather than beginning with the scattering amplitude we immediately turn to the squared matrix elements, averaged (summed) over the helicities and color of incoming (outgoing) quarks. The polarization vectors of the photon are

$$
\begin{aligned}
\varepsilon_{L} & =\frac{1}{Q}\left(q^{\prime}+x p\right), \\
\varepsilon_{ \pm} & =\frac{1}{\sqrt{2}}(0,1, \pm i, 0) .
\end{aligned}
$$

In fact, since $\varepsilon_{L}=q / Q+2 x p / Q$, we make use of gauge invariance and simply put $\varepsilon_{L}=$ $2 x p / Q$. In this paper we restrict ourselves to the longitudinal polarization; the transverse case will be presented in a subsequent paper.

Our calculations are performed in the Feynman gauge. As to the internal ( $t$-channel) gluon numerators, we use the decomposition

$$
g_{\mu \nu}=\frac{2}{s}\left(p_{\mu} q_{\nu}^{\prime}+p_{\nu} q_{\mu}^{\prime}\right)+g_{\mu \nu}^{\perp} .
$$

In the high energy limit we retain only the first term, since the contributions of the other terms will be suppressed by powers of $t / s$. For the diagrams where the produced gluon couples to the lower fermion line, this may not be immediately obvious, since some of the light cone components of the $t$ channel gluon are not small; we have checked the approximation against a calculation involving the full numerator, and we have obtained the same answer. In the diagrams $A_{5}$ and $B_{5}$ we have combined two Feynman graphs (Fig. (1) into one effective diagram. Since the produced gluon is far from the lower quark in rapidity, we can simplify the emission vertex: 


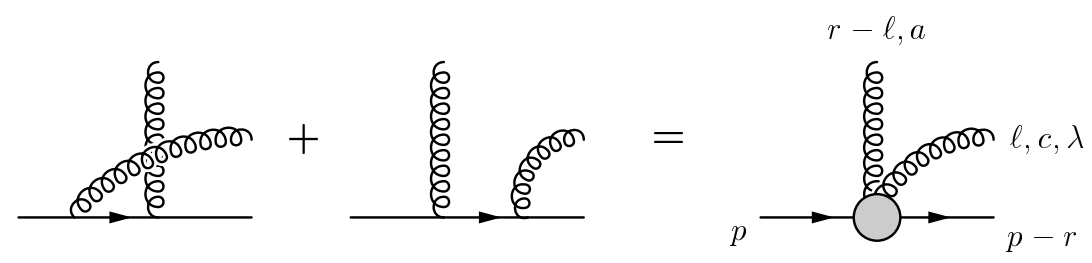

FIG. 4. Emission of a gluon from the lower quark line.

$$
\bar{u}\left(p-r, h^{\prime}\right)\left[\frac{\not \prime^{\prime}(\not p-\ell) \gamma^{\lambda}}{(p-\ell)^{2}} t^{a} t^{c}+\frac{\gamma^{\lambda}\left(\not p+\not l-\gamma^{\prime}\right) \not^{\prime}}{(p+\ell-r)^{2}} t^{c} t^{a}\right] u(p, h) \approx \frac{2 p^{\lambda}}{\alpha_{\ell}}\left[t^{c}, t^{a}\right] \delta_{h h^{\prime}} .
$$

Here we have used that $\ell$ has large components only in the $q^{\prime}$ direction; $h$ and $h^{\prime}$ denote the helicities of the lower incoming and outgoing quarks, respectively, and the Lorentz index $\lambda$ is to be contracted with the polarization vector of the outgoing gluon with color index c. $t^{a}$ and $t^{c}$ are the color group generators in the fundamental representation, and $a$ denotes the color index carried by the $t$-channel gluon.

The result for the helicity-summed square of the matrix element (averaged over helicity and color of the incoming quark) has the following form:

$$
|\mathcal{M}|^{2}=e^{2} e_{f}^{2} g^{4} \delta^{a b} 16 Q^{2} s^{2} \sum_{i, j=1}^{5}\left(\mathcal{A A}_{i j}+\mathcal{A B}_{i j}+\mathcal{B A}_{i j}+\mathcal{B B}_{i j}\right) \frac{1}{\boldsymbol{r}^{4}} \frac{g^{2} \delta^{a b}}{2 N_{c}},
$$

where $e e_{f}$ is the charge of quark flavour $f$, and $g$ is the strong coupling constant. Following the labeling of Fig. 3 we denote the product of graph $A_{i}$ with graph $A_{j}$ by $\mathcal{A A}_{i j}$ etc. Furthermore, the phase space over the final state (including the $\delta$-functions from the onshell conditions) is given by:

$$
d \Phi_{4}=\frac{d \alpha d \alpha_{\ell} d^{D-2} \boldsymbol{k} d^{D-2} \boldsymbol{\ell} d^{D-2} \boldsymbol{r}}{8 s(2 \pi)^{3 D-4} \alpha_{1} \bar{\alpha}_{1} \alpha_{\ell}} .
$$

$D$ denotes the space time dimension. We calculate our results in dimensional regularization with transverse dimension $D-2=2-2 \epsilon$.

The sum on the rhs of (32), together with the phase space (33) and the flux factor $1 /(2 s)$ represents the (helicity-summed) cross section of the process $\gamma^{*}+q \rightarrow(q \bar{q} g)+q$ :

$$
d \sigma=\frac{1}{2 s}|\mathcal{M}|^{2} d \Phi_{4}
$$

The NLO real corrections to the photon impact factor will be obtained from the sum on the rhs of (32) by doing the integral over the $q \bar{q} g$ phase space and invariant mass. However, we will have to subtract the production of the gluon in the central region (multiregge kinematics). This piece belongs to the LO BFKL calculation and must not be counted twice (see below). Integrating over the momentum of the outgoing gluon leads to infrared singularities which cancel against the infrared singularities of the virtual corrections [9]. Since the calculation of the virtual corrections has been done using the dimensional regularization, we will use these same techniques also for the real corrections.

In order to obtain the NLO jet cross section for the off-shell photon-gluon fusion process $\gamma^{*}+g \rightarrow(2$ jets, 3 jets), we have to combine the sum in (32) with the virtual NLO corrections to $\gamma^{*}+g \rightarrow q \bar{q}$, using a suitable jet definition. 


\section{RESULTS}

In this section we list our results for the helicity-summed matrix element. The traces have been calculated with the help of the Mathematica package FeynCalc [10]. The results have then been simplified further by casting them into a factorizing form: in order to have the photon wave function interpretation we have tried to find a representation in which each term $\mathcal{A A}_{i j}$ can be written as a sum of products of two terms: one factor belongs to the incoming photon, the other one to the outgoing photon, and in between we have a piece which belongs to the cross section which describes the interaction of the $q \bar{q} g$-system with the lower quark. After some algebra we arrive at the following results:

$$
\begin{aligned}
\mathcal{A A}_{11}= & C_{F} \frac{2}{D_{1}^{2} D_{2}^{2}}(1-\epsilon) \alpha_{1} \bar{\alpha}_{1} \boldsymbol{A}_{1}^{2} \\
\mathcal{A A}_{12}= & -\frac{1}{N_{c}} \frac{1}{D_{1} D_{2}^{2} D_{4}}\left((1-\epsilon) \alpha_{1} \alpha_{2} \boldsymbol{A}_{1} \boldsymbol{A}_{2}-\alpha_{1} \alpha_{2}^{2} \bar{\alpha}_{1} \boldsymbol{r}^{2}\right) \\
\mathcal{A}_{13}= & C_{F} \frac{2}{D_{1}^{2} D_{2} D_{3}}\left((1-\epsilon) \bar{\alpha}_{1}^{2} \boldsymbol{A}_{1} \boldsymbol{A}_{3}+\bar{\alpha}_{1}^{2}(\boldsymbol{k}+\boldsymbol{r})(\boldsymbol{k}+\boldsymbol{\ell}+\boldsymbol{r})\right) \\
\mathcal{A A}_{14}= & -N_{c} \frac{1}{D_{1} D_{2}^{2} D_{5}}\left(\frac{1}{2 \alpha_{\ell}} \alpha_{1}\left(\alpha_{2} \bar{\alpha}_{1}+2(1-\epsilon) \alpha_{\ell}^{2}\right) \boldsymbol{A}_{1} \boldsymbol{A}_{4}\right. \\
& \left.+\frac{1}{2} \alpha_{1} \alpha_{2} \bar{\alpha}_{1}^{2} D_{2}+\frac{1}{2 \alpha_{\ell}} \alpha_{1} \alpha_{2}^{2} \bar{\alpha}_{1} \boldsymbol{A}_{1} \boldsymbol{r}-\alpha_{1} \alpha_{2}^{2} \bar{\alpha}_{1} \boldsymbol{r}^{2}\right) \\
\mathcal{A A}_{15}= & N_{c} \frac{1}{\alpha_{\ell} D_{1} D_{2}^{2} D_{5}} \alpha_{1} \alpha_{2}^{2} \bar{\alpha}_{1}^{2} \boldsymbol{r}^{2} \\
\mathcal{A A}_{22}= & C_{F} \frac{2}{\bar{\alpha}_{1} D_{2}^{2} D_{4}^{2}}(1-\epsilon) \alpha_{1} \alpha_{2}^{2} \boldsymbol{A}_{2}^{2} \\
\mathcal{A A}_{23}= & -\frac{1}{N_{c}} \frac{1}{D_{1} D_{2} D_{3} D_{4}}\left((1-\epsilon) \alpha_{2} \bar{\alpha}_{1} \boldsymbol{A}_{2} \boldsymbol{A}_{3}+\alpha_{2} \bar{\alpha}_{1}\left(\boldsymbol{k}+\boldsymbol{r}-\alpha_{1} \boldsymbol{r}\right)(\boldsymbol{k}+\boldsymbol{\ell}\right. \\
\mathcal{A A}_{24}= & N_{c} \frac{1}{D_{2}^{2} D_{4} D_{5}}\left(\frac{1}{2 \alpha_{\ell} \bar{\alpha}_{1}} \alpha_{1} \alpha_{2}\left(\alpha_{2}\right)\right) \\
& \left.+\frac{1}{2} \alpha_{1} \alpha_{2}^{2} \bar{\alpha}_{1} D_{2}+\frac{1}{2 \alpha_{\ell}} \alpha_{1} \alpha_{2}^{2} \bar{\alpha}_{1} \boldsymbol{A}_{2} \boldsymbol{r}+\alpha_{1} \alpha_{2}^{2} \bar{\alpha}_{1} \boldsymbol{r}^{2}\right) \\
& \left.+\frac{3}{2} \alpha_{2} \bar{\alpha}_{1} \boldsymbol{A}_{3}(\boldsymbol{k}+\boldsymbol{\ell})\right) \\
\mathcal{A A}_{25}= & -N_{c} \frac{1}{\alpha_{\ell} D_{2}^{2} D_{4} D_{5}} \alpha_{1} \alpha_{2}^{3} \bar{\alpha}_{1} \boldsymbol{r}^{2} \\
\mathcal{A A}_{33}= & C_{F} \frac{2}{\alpha_{1} D_{1}^{2} D_{3}^{2}}(1-\epsilon) \bar{\alpha}_{1}^{3} \boldsymbol{A}_{3}^{2} \\
\mathcal{A A}_{34}= & N_{c} \frac{1}{D_{1} D_{2} D_{3} D_{5}}\left(\frac{1}{2 \alpha_{\ell}} \bar{\alpha}_{1}\left(\alpha_{1} \bar{\alpha}_{1}-\alpha_{\ell}-2(1-\epsilon) \alpha_{\ell}^{2}\right) \boldsymbol{A}_{3} \boldsymbol{A}_{4}\right. \\
& -\frac{1}{2} \alpha_{1} \bar{\alpha}_{1}^{2} \bar{\alpha}_{2} D_{2}+\alpha_{1} \alpha_{2} \bar{\alpha}_{1}^{2} D_{7}+\frac{1}{2 \alpha_{\ell}} \alpha_{1} \alpha_{2} \bar{\alpha}_{1}^{2} \boldsymbol{A}_{3} \boldsymbol{r}+\alpha_{1} \alpha_{2} \bar{\alpha}_{1}^{2} \boldsymbol{r}^{2} \\
&
\end{aligned}
$$




$$
\begin{aligned}
& \mathcal{A A}_{35}=-N_{c} \frac{1}{\alpha_{\ell} D_{1} D_{2} D_{3} D_{5}} \alpha_{1} \alpha_{2} \bar{\alpha}_{1}^{2} \bar{\alpha}_{2} \boldsymbol{r}^{2} \\
& \mathcal{A A}_{44}=N_{c} \frac{1}{D_{2}^{2} D_{5}^{2}}\left(\frac{2}{\bar{\alpha}_{1}} \boldsymbol{A}_{4}^{2} \alpha_{1}\left((1-\epsilon) \alpha_{\ell}^{2}+\alpha_{2} \bar{\alpha}_{1}\right)+\alpha_{1} \alpha_{2} \alpha_{\ell} \bar{\alpha}_{1} D_{2}+2 \alpha_{1} \alpha_{2}^{2} \bar{\alpha}_{1} \boldsymbol{r}^{2}\right) \\
& \mathcal{A A}_{45}=-N_{c} \frac{1}{D_{2}^{2} D_{5}^{2}} \alpha_{1} \alpha_{2}^{2} \bar{\alpha}_{1} \boldsymbol{r}^{2} \\
& \mathcal{A} \mathcal{A}_{55}=0 \\
& \mathcal{A B}_{11}=\frac{1}{N_{c}} \frac{1}{D_{1} D_{2} D_{6} D_{7}}\left((1-\epsilon) \alpha_{1} \bar{\alpha}_{1} \boldsymbol{A}_{1} \boldsymbol{B}_{1}+\alpha_{1} \bar{\alpha}_{1}\left(\boldsymbol{k}+\alpha_{1} \boldsymbol{r}\right)\left(\boldsymbol{k}+\boldsymbol{\ell}+\bar{\alpha}_{2} \boldsymbol{r}\right)\right) \\
& \mathcal{A B}_{12}=-C_{F} \frac{2}{D_{1} D_{2} D_{3} D_{7}}\left((1-\epsilon) \bar{\alpha}_{1} \bar{\alpha}_{2} \boldsymbol{A}_{1} \boldsymbol{B}_{2}+\bar{\alpha}_{1} \bar{\alpha}_{2}(\boldsymbol{k}+\boldsymbol{r})(\boldsymbol{k}+\boldsymbol{\ell}+\boldsymbol{r})\right) \\
& \mathcal{A B}_{13}=\frac{1}{N_{c}} \frac{1}{D_{1} D_{2} D_{4} D_{6}}\left((1-\epsilon) \alpha_{1}^{2} \boldsymbol{A}_{1} \boldsymbol{B}_{3}-\alpha_{1}^{2} \alpha_{2} \bar{\alpha}_{1} \boldsymbol{r}^{2}\right) \\
& \mathcal{A B}_{14}=N_{c} \frac{1}{D_{1} D_{2} D_{5} D_{7}}\left(\frac{1}{2 \alpha_{\ell}} \bar{\alpha}_{1}\left(\alpha_{1} \bar{\alpha}_{1}-\alpha_{\ell}-(1-\epsilon) 2 \alpha_{\ell}^{2}\right) \boldsymbol{A}_{1} \boldsymbol{B}_{4}\right. \\
& +\alpha_{1} \bar{\alpha}_{1}^{2} \bar{\alpha}_{2} D_{2}-\frac{1}{2} \alpha_{1} \alpha_{2} \bar{\alpha}_{1}^{2} D_{7}+\frac{1}{2 \alpha_{\ell}} \alpha_{1} \alpha_{2} \bar{\alpha}_{1} \bar{\alpha}_{2} \boldsymbol{A}_{1} \boldsymbol{r}-\alpha_{1} \alpha_{2} \bar{\alpha}_{1} \bar{\alpha}_{2} \boldsymbol{r}^{2} \\
& \left.-\frac{3}{2} \bar{\alpha}_{1} \bar{\alpha}_{2} \boldsymbol{A}_{1}(\boldsymbol{k}+\boldsymbol{r})\right) \\
& \mathcal{A B}_{15}=-N_{c} \frac{1}{\alpha_{\ell} D_{1} D_{2} D_{5} D_{7}} \alpha_{1} \alpha_{2} \bar{\alpha}_{1}^{2} \bar{\alpha}_{2} \boldsymbol{r}^{2} \\
& \mathcal{A B}_{22}=\frac{1}{N_{c}} \frac{1}{D_{2} D_{3} D_{4} D_{7}}\left((1-\epsilon) \alpha_{2} \bar{\alpha}_{2} \boldsymbol{A}_{2} \boldsymbol{B}_{2}+\alpha_{2} \bar{\alpha}_{2}\left(\boldsymbol{k}+\boldsymbol{r}-\alpha_{1} \boldsymbol{r}\right)\left(\boldsymbol{k}+\boldsymbol{\ell}+\bar{\alpha}_{1} \boldsymbol{r}\right)\right) \\
& \mathcal{A B}_{23}=-C_{F} \frac{2}{\bar{\alpha}_{1} D_{2} D_{4}^{2} D_{6}}(1-\epsilon) \alpha_{1}^{2} \alpha_{2} \boldsymbol{A}_{2} \boldsymbol{B}_{3} \\
& \mathcal{A B}_{24}=-N_{c} \frac{1}{D_{2} D_{4} D_{5} D_{7}}\left(\frac{1}{2 \alpha_{\ell}} \alpha_{2}\left(\alpha_{1} \bar{\alpha}_{1}-\alpha_{\ell}-2(1-\epsilon) \alpha_{\ell}^{2}\right) \boldsymbol{A}_{2} \boldsymbol{B}_{4}\right. \\
& +\alpha_{1} \alpha_{2} \bar{\alpha}_{1} \bar{\alpha}_{2} D_{2}-\frac{1}{2} \alpha_{1} \alpha_{2}^{2} \bar{\alpha}_{1} D_{7}+\frac{1}{2 \alpha_{\ell}} \alpha_{1} \alpha_{2} \bar{\alpha}_{1} \bar{\alpha}_{2} \boldsymbol{A}_{2} \boldsymbol{r}+\alpha_{1} \alpha_{2} \bar{\alpha}_{1} \bar{\alpha}_{2} \boldsymbol{r}^{2} \\
& \left.-\frac{3}{2} \alpha_{2} \bar{\alpha}_{2} \boldsymbol{A}_{2}(\boldsymbol{k}+\boldsymbol{r})\right) \\
& \mathcal{A B}_{25}=N_{c} \frac{1}{\alpha_{\ell} D_{2} D_{4} D_{5} D_{7}} \alpha_{1} \alpha_{2}^{2} \bar{\alpha}_{1} \bar{\alpha}_{2} \boldsymbol{r}^{2} \\
& \mathcal{A B}_{33}=\frac{1}{N_{c}} \frac{1}{D_{1} D_{3} D_{4} D_{6}}\left((1-\epsilon) \alpha_{1} \bar{\alpha}_{1} \boldsymbol{A}_{3} \boldsymbol{B}_{3}+\alpha_{1} \bar{\alpha}_{1}\left(\boldsymbol{k}+\boldsymbol{r}-\alpha_{1} \boldsymbol{r}\right)\left(\boldsymbol{k}+\boldsymbol{\ell}+\bar{\alpha}_{1} \boldsymbol{r}\right)\right) \\
& \mathcal{A B}_{34}=-N_{c} \frac{1}{D_{1} D_{3} D_{5} D_{7}}\left(\frac{1}{2 \alpha_{1} \alpha_{\ell}} \bar{\alpha}_{1}^{2}\left(\alpha_{1} \bar{\alpha}_{2}+2(1-\epsilon) \alpha_{\ell}^{2}\right) \boldsymbol{A}_{3} \boldsymbol{B}_{4}\right. \\
& \left.+\frac{1}{2} \alpha_{1} \bar{\alpha}_{1}^{2} \bar{\alpha}_{2} D_{7}+\frac{1}{2 \alpha_{\ell}} \alpha_{1} \bar{\alpha}_{1}^{2} \bar{\alpha}_{2} \boldsymbol{A}_{3} \boldsymbol{r}+\alpha_{1} \bar{\alpha}_{1}^{2} \bar{\alpha}_{2} \boldsymbol{r}^{2}\right) \\
& \mathcal{A B}_{35}=N_{c} \frac{1}{\alpha_{\ell} D_{1} D_{3} D_{5} D_{7}} \alpha_{1} \bar{\alpha}_{1}^{2} \bar{\alpha}_{2}^{2} \boldsymbol{r}^{2}
\end{aligned}
$$




$$
\begin{aligned}
\mathcal{A B}_{44}= & -N_{c} \frac{1}{D_{2} D_{5}^{2} D_{7}}\left(\left(2 \alpha_{1} \bar{\alpha}_{1}+\alpha_{\ell}-2(1-\epsilon) \alpha_{\ell}^{2}\right) \boldsymbol{A}_{4} \boldsymbol{B}_{4}\right. \\
& \left.+\frac{1}{2} \alpha_{1} \alpha_{\ell} \bar{\alpha}_{1} \bar{\alpha}_{2} D_{2}+\frac{1}{2} \alpha_{1} \alpha_{2} \alpha_{\ell} \bar{\alpha}_{1} D_{7}+2 \alpha_{1} \alpha_{2} \bar{\alpha}_{1} \bar{\alpha}_{2} \boldsymbol{r}^{2}-\alpha_{\ell}^{2}(\boldsymbol{k}+\boldsymbol{\ell})(\boldsymbol{k}+\boldsymbol{r})\right) \\
\mathcal{A B}_{45}= & N_{c} \frac{1}{D_{2} D_{5}^{2} D_{7}} \alpha_{1} \alpha_{2} \bar{\alpha}_{1} \bar{\alpha}_{2} \boldsymbol{r}^{2} \\
\mathcal{A B}_{55}= & 0 .
\end{aligned}
$$

Here we have used the following abbreviations:

$$
\begin{aligned}
& \boldsymbol{A}_{1}=\alpha_{2} \boldsymbol{\ell}+\alpha_{\ell}(\boldsymbol{k}+\boldsymbol{r}) \\
& \boldsymbol{A}_{2}=\bar{\alpha}_{1} \boldsymbol{\ell}+\alpha_{\ell}(\boldsymbol{k}+\boldsymbol{\ell}) \\
& \boldsymbol{A}_{3}=\alpha_{1} \boldsymbol{\ell}-\alpha_{\ell}(\boldsymbol{k}+\boldsymbol{r}) \\
& \boldsymbol{A}_{4}=\alpha_{2}(\boldsymbol{\ell}-\boldsymbol{r})+\alpha_{\ell}(\boldsymbol{k}+\boldsymbol{r}) \\
& \boldsymbol{B}_{1}=\bar{\alpha}_{2} \boldsymbol{\ell}-\alpha_{\ell}(\boldsymbol{k}+\boldsymbol{\ell}) \\
& \boldsymbol{B}_{2}=\alpha_{1} \boldsymbol{\ell}-\alpha_{\ell}(\boldsymbol{k}+\boldsymbol{r}) \\
& \boldsymbol{B}_{3}=\bar{\alpha}_{1} \boldsymbol{\ell}+\alpha_{\ell}(\boldsymbol{k}+\boldsymbol{\ell}) \\
& \boldsymbol{B}_{4}=\bar{\alpha}_{2}(\boldsymbol{\ell}-\boldsymbol{r})-\alpha_{\ell}(\boldsymbol{k}+\boldsymbol{\ell}) .
\end{aligned}
$$

In the above definitions, the vectors $\boldsymbol{B}_{i}$ are obtained from the $\boldsymbol{A}_{i}$ by the transformations (11), (12). Note also the additional relations

$$
\boldsymbol{A}_{2}=\boldsymbol{B}_{3}, \quad \boldsymbol{A}_{3}=\boldsymbol{B}_{2} .
$$

The remaining terms $\mathcal{A A}_{i j}$ (with $i>j$ ) and $\mathcal{B A}_{i j}$ (with $i>j$ ) follow from the symmetry properties $\mathcal{A A}_{i j}=\mathcal{A A}_{j i}$ and $\mathcal{A B}_{i j}=\mathcal{B A}_{j i}$; in order to find $\mathcal{B A}_{i j}$ and $\mathcal{A B}_{j i}$ (with $i<j$ ), and all the $\mathcal{B B}_{i j}$ 's we use the substitutions (11), (12).

We finally note that in the sum of the four $(i j)=(44)$ matrix elements: $\mathcal{A A}_{44}+\mathcal{A B}_{44}+$ $\mathcal{B A}_{44}+\mathcal{B B}_{44}$, the term proportional to $D_{2}$ in $\mathcal{A A}_{44}$, the terms proportional to $D_{2}$ and $D_{7}$ in $\mathcal{A B}_{44}$, and the analogous terms in $\mathcal{B A}_{44}$ and $\mathcal{B B}_{44}$ cancel against each other.

An important feature of the results (35)-(64) is the ultraviolet behavior of transverse momenta: individual terms, namely $\mathcal{A A}_{11}$ and $\mathcal{A A}_{22}, \mathcal{A A}_{12}$ and $\mathcal{A A}_{21}$, and $\mathcal{A A}_{14}, \mathcal{A A}_{24}$, $\mathcal{A A}_{41}, \mathcal{A A}_{42}$, and $\mathcal{A A}_{44}$ go as $1 / \boldsymbol{\ell}^{2}$ for $\boldsymbol{\ell}^{2} \gg \boldsymbol{k}^{2}$. When integrating over $\boldsymbol{\ell}$, they lead to logarithmic divergencies. However, in the sum these divergencies cancel, and all transverse momentum integrals are ultraviolet finite.

\section{THE CENTRAL REGION AND THE ENERGY SCALE}

As we have discussed in the beginning of section II, our results are valid in the fragmentation

region of the photon and in the 'upper' part of the central region. Before we move on to any further evaluation of our results we have to address the question of how to remove the 
central region. Let us, therefore, first study the limit where the emitted gluon belongs to the central rapidity region:

$$
\begin{gathered}
\frac{|\ell|}{\sqrt{s}} \leq \alpha_{\ell} \leq \alpha_{\ell 0} \\
\frac{\ell^{2}}{s \alpha_{\ell 0}} \leq \beta_{\ell} \leq \frac{|\ell|}{\sqrt{s}} .
\end{gathered}
$$

In this limit we should find agreement with the leading logarithmic approximation. First of all, we note that the phase space integral in (33) contains a factor $1 / \alpha_{\ell}$ : this makes the $\alpha_{\ell}$ integral divergent, since, as we will see, some of the $\mathcal{A A}_{i j}$ have a finite but non vanishing limit for $\alpha_{\ell} \rightarrow 0$. Let us see how this works in detail. In the limit of small $\alpha_{\ell}$, the denominators $D_{1}, \ldots, D_{7}((17)-(23))$ are expressed in terms of the two denominators $D_{2}$ and $D_{7}$; they in turn are closely related to the denominators $D(\boldsymbol{k}+\boldsymbol{r})=\alpha(1-\alpha) Q^{2}+(\boldsymbol{k}+\boldsymbol{r})^{2}$ and $D(\boldsymbol{k}+\boldsymbol{\ell})=\alpha(1-\alpha) Q^{2}+(\boldsymbol{k}+\boldsymbol{\ell})^{2}$, that are well known from the Born approximation:

$$
\begin{aligned}
& D_{1} \rightarrow-\frac{1-\alpha}{\alpha_{\ell}} \ell^{2}, \\
& D_{2} \rightarrow-\frac{1}{\alpha} D(\boldsymbol{k}+\boldsymbol{r}), \\
& D_{3} \rightarrow \frac{\alpha}{\alpha_{\ell}} \ell^{2}, \\
& D_{4} \rightarrow \frac{1-\alpha}{\alpha_{\ell}} \ell^{2}, \\
& D_{5} \rightarrow-(\boldsymbol{\ell}-\boldsymbol{r})^{2}, \\
& D_{6} \rightarrow-\frac{\alpha}{\alpha_{\ell}} \ell^{2}, \\
& D_{7} \rightarrow-\frac{1}{1-\alpha} D(\boldsymbol{k}+\boldsymbol{\ell}) .
\end{aligned}
$$

In the numerators on the rhs of (35)-(64) we expand in powers of $\alpha_{\ell}$ and keep only the leading term. The contributions from the diagrams containing $A_{3}$ or $B_{3}$ drop out completely, since they involve an extra fermion propagator that gives a suppression by a power of $\alpha_{\ell}$. Similarly the diagrams with $\mathcal{A}_{1}, \mathcal{A}_{2}$ (or $\mathcal{B}_{1}, \mathcal{B}_{2}$ ) on both sides. Nonzero contributions come from diagrams involving $\mathcal{A}_{4}$ or $\mathcal{B}_{4}$, but they cancel in the sum. We are finally left with the contributions coming from $\mathcal{A}_{5}$ and $\mathcal{B}_{5}$. As to the $\mathcal{A}_{\mathcal{A}_{i j}}$-terms, all non vanishing terms have the common factor $\frac{1}{\left(D_{2}\right)^{2}}$; all non vanishing $\mathcal{A B}_{i j}$ terms contain $\frac{1}{D_{2} D_{7}}$. For the sum on the rhs of (32) we find the simplified form

$$
|\mathcal{M}|^{2}=\frac{2 s^{2}}{\boldsymbol{r}^{4}} \frac{2(2 \pi)^{3}}{3} g^{6} \frac{\delta^{a b}}{2 N_{c}} N_{c} \delta^{a b} \frac{\alpha(1-\alpha)\left|\Psi_{L}(\boldsymbol{k}+\boldsymbol{\ell}, \alpha)\right|^{2}}{(\boldsymbol{\ell}-\boldsymbol{r})^{2}} \sum_{i, j=1}^{5} \mathcal{D}_{i j}
$$

Here,

$$
\left|\Psi_{L}(\boldsymbol{k}+\boldsymbol{\ell}, \alpha)\right|^{2}=\frac{6 \alpha_{\mathrm{em}} e_{f}^{2}}{\pi^{2}} \alpha^{2}(1-\alpha)^{2} Q^{2}\left(\frac{1}{D(\boldsymbol{k}+\boldsymbol{r})}-\frac{1}{D(\boldsymbol{k}+\boldsymbol{\ell})}\right)^{2}
$$


is the square of the leading order (longitudinal) photon wave function. The matrix $\mathcal{D}_{i j}$ does not discriminate between the coupling of the $t$-channel gluon to the upper quark or antiquark lines, i.e. the difference between $\mathcal{A}_{i}$ and $\mathcal{B}_{i}$ is contained entirely inside the photon wave function. The matrix itself has the rather simple form

$$
\left(\begin{array}{ccccc}
0 & 0 & 0 & -\frac{1}{2} & \frac{\boldsymbol{r}^{2}}{\boldsymbol{\ell}^{2}} \\
0 & 0 & 0 & -\frac{1}{2} & \frac{\boldsymbol{r}^{2}}{\boldsymbol{\ell}^{2}} \\
0 & 0 & 0 & 0 & 0 \\
-\frac{1}{2} & -\frac{1}{2} & 0 & 2+2 \frac{\boldsymbol{r}^{2}}{(\boldsymbol{\ell}-\boldsymbol{r})^{2}} & -\frac{\boldsymbol{r}^{2}}{(\boldsymbol{\ell}-\boldsymbol{r})^{2}} \\
\frac{\boldsymbol{r}^{2}}{\boldsymbol{\ell}^{2}} & \frac{\boldsymbol{r}^{2}}{\boldsymbol{\ell}^{2}} & 0 & -\frac{\boldsymbol{r}^{2}}{(\boldsymbol{\ell}-\boldsymbol{r})^{2}} & 0
\end{array}\right)
$$

The sum of all diagrams is then given by

$$
|\mathcal{M}|^{2}=2 s^{2} \frac{2(2 \pi)^{3}}{3} g^{4} \delta^{a b} \alpha(1-\alpha)\left|\Psi_{L}(\boldsymbol{k}+\boldsymbol{\ell}, \alpha)\right|^{2} \frac{1}{(\boldsymbol{\ell}-\boldsymbol{r})^{4}} \frac{4 N_{c} \boldsymbol{r}^{2}(\boldsymbol{\ell}-\boldsymbol{r})^{2}}{\boldsymbol{\ell}^{2}} \frac{1}{\boldsymbol{r}^{4}} \frac{g^{2} \delta^{a b}}{2 N_{c}}
$$

This is exactly what we expect: the factor $\alpha(1-\alpha)\left|\Psi_{L}\right|^{2}$, including the color factor $\delta^{a b}$ gives the impact factor for virtual photons in the LLA. The factors $1 /(\boldsymbol{r}-\boldsymbol{\ell})^{4}$ and $1 / \boldsymbol{r}^{4}$ are the propagators of the reggeized gluons in the $t$-channel, and $4 N_{c} \boldsymbol{r}^{2}(\boldsymbol{r}-\boldsymbol{\ell})^{2} / \boldsymbol{\ell}^{2}$ is the square of the Lipatov vertex [11]. Finally, $\delta^{a b} /\left(2 N_{c}\right)$ is the LLA quark impact factor (averaged (summed) over initial (final) colors and helicities). Together with the $\alpha_{\ell}$ integral in the region (74) which gives a ln- $s$ factor, we recover the LO BFKL result. This demonstrates that our results correctly reproduce the central region of the produced gluon. It may be interesting to note that (79) may also be written in the factorized form:

$$
\begin{aligned}
|\mathcal{M}|^{2}= & 2 s^{2} \frac{2(2 \pi)^{3}}{3} g^{4} \delta^{a b} \alpha(1-\alpha)\left|\Psi_{L}(\boldsymbol{k}+\boldsymbol{\ell}, \alpha)\right|^{2} \\
& \times 4 N_{c}\left[\frac{\boldsymbol{r}-\boldsymbol{\ell}}{(\boldsymbol{r}-\boldsymbol{\ell})^{2}}+\frac{\boldsymbol{\ell}}{\boldsymbol{\ell}^{2}}\right] \cdot\left[\frac{\boldsymbol{r}-\boldsymbol{\ell}}{(\boldsymbol{r}-\boldsymbol{\ell})^{2}}+\frac{\boldsymbol{\ell}}{\boldsymbol{\ell}^{2}}\right] \frac{1}{\boldsymbol{r}^{4}} \frac{g^{2} \delta^{a b}}{2 N_{c}} .
\end{aligned}
$$

In this way it has the same structure as (35)-(64): a product of two terms, one belonging to the incoming photon, one to the outgoing.

In order to avoid double counting we have to remove this central region from our results. Details of this subtraction procedure will be presented elsewhere, here we only sketch the general argument 12, 13]. Consider, as an example, the contribution $\mathcal{A A}_{15}$ which, as $\alpha_{\ell} \rightarrow 0$, has a non vanishing limit. The integral over $\alpha_{\ell}$ diverges logarithmically, but the ln $s$-piece belongs to the LO BFKL calculation. We therefore decompose:

$$
\begin{aligned}
& \int_{\frac{|l|}{\sqrt{s}}}^{1-\alpha} \frac{d \alpha_{\ell}}{\alpha_{\ell}} \mathcal{A A}_{15}\left(\alpha_{\ell}\right)= \\
& \int_{\frac{|l|}{\sqrt{s}}}^{1-\alpha} \frac{d \alpha_{\ell}}{\alpha_{\ell}}\left[\mathcal{A A}_{15}\left(\alpha_{\ell}\right)-\mathcal{A A}_{15}(0) \theta\left(\alpha_{\ell 0}-\alpha_{\ell}\right)\right]+\mathcal{A A}_{15}(0) \ln \left(\sqrt{s} \frac{\alpha_{\ell 0}}{|\ell|}\right),
\end{aligned}
$$

where $\alpha_{\ell 0}$ denotes an energy independent scale much smaller than unity which may depend upon the other momenta, e.g. $\boldsymbol{\ell}$. As an example, we could define, as the boundary between 
the fragmentation region of the photon and the central region, a cutoff on the invariant mass of the $q \bar{q} g$ system, $M_{0}^{2}$, which we choose to be larger than $Q^{2}, \boldsymbol{\ell}^{2}, \boldsymbol{k}^{2}$ and $\boldsymbol{r}^{2}$. In this case $\alpha_{\ell 0} \approx \frac{\ell^{2}}{M_{0}^{2}}$. In the first term on the rhs, we can safely put to zero the lower limit of integration (the error is of order $1 / \sqrt{s}$ ), the second term is part of the LO BFKL calculation, now with a definite scale inside the logarithm. This example illustrates the rôle of the energy scale within the result of the NLO calculation: a change in the scale results in a finite term. As an example, starting from (81) and adding the analogous contribution from the 'lower' part of the central region (where we have an analogous cutoff parameter $\beta_{\ell 0}$ ), we can write:

$$
\ln \left(\sqrt{s} \frac{\alpha_{\ell 0}}{|\boldsymbol{\ell}|}\right)+\ln \left(\sqrt{s} \frac{\beta_{\ell 0}}{|\boldsymbol{\ell}|}\right)=\ln \left(\frac{s}{|\boldsymbol{r}||\boldsymbol{r}-\boldsymbol{\ell}|}\right)+\ln \left(\frac{|\boldsymbol{r}| \boldsymbol{r}-\boldsymbol{\ell} \mid \alpha_{\ell 0} \beta_{\ell 0}}{\boldsymbol{\ell}^{2}}\right) .
$$

From this we see that our NLO correction does not fix the energy scale, but, for a given choice of the scale, it provides a well-defined answer. Moreover, it then determines how this answer changes under a modification of the energy scale.

This feature becomes more transparent if we generalize to infinite order in $\alpha_{s}$. Let us consider, instead of the process $\gamma^{*} q \rightarrow(q \bar{q} g) q$, the scattering of two virtual photons with virtualities $Q_{1}^{2}$ and $Q_{2}^{2}$, i.e. we replace the lower quark line by another photon impact factor. In the LO approximation, the scale of the energy factor in the total cross section is not fixed, i.e. any change of the scale exceeds the LO accuracy. One possible choice is the symmetric scale $s_{0}=k k^{\prime}, k=|\boldsymbol{k}|, k^{\prime}=\left|\boldsymbol{k}^{\prime}\right|$ :

$$
\begin{aligned}
\sigma_{\gamma^{*} \gamma^{*}}(s)= & \frac{1}{(2 \pi)^{2}} \int_{\delta-i \infty}^{\delta+i \infty} \frac{d \omega}{2 \pi i} \int \frac{d^{2} \boldsymbol{k}}{\boldsymbol{k}^{2}} \int \frac{d^{2} \boldsymbol{k}^{\prime}}{\boldsymbol{k}^{\prime 2}} \\
& \times\left(\frac{s}{k k^{\prime}}\right)^{\omega} \Phi_{\gamma^{*}}\left(\boldsymbol{k}, Q_{1}\right) \mathcal{G}_{\omega}\left(\boldsymbol{k}, \boldsymbol{k}^{\prime}\right) \Phi_{\gamma^{*}}\left(\boldsymbol{k}^{\prime}, Q_{2}\right) .
\end{aligned}
$$

This choice seems to be most natural from the point of view of the Green's function. On the other hand, for a high energy scattering amplitude it is more natural to chose the 'physical' scale $s_{0}=\sqrt{Q_{1}^{2} Q_{2}^{2}}$. In NLO, a change of the scale affects both the impact factors and the Green's function, i.e. the definition of these quantities becomes scale dependent. For example, starting from (83) where all elements are defined with respect to the scale $\left(k k^{\prime}\right)$, and changing to the 'physical' scale $\sqrt{Q_{1}^{2} Q_{2}^{2}}$ we obtain:

$$
\begin{aligned}
\sigma_{\gamma^{*} \gamma^{*}}(s)= & \frac{1}{(2 \pi)^{2}} \int_{\delta-i \infty}^{\delta+i \infty} \frac{d \omega}{2 \pi i} \int \frac{d^{2} \boldsymbol{k}}{\boldsymbol{k}^{2}} \int \frac{d^{2} \boldsymbol{k}^{\prime}}{\boldsymbol{k}^{\prime 2}} \\
& \times\left(\frac{s}{Q_{1} Q_{2}}\right)^{\omega} \Phi_{\gamma^{*}}\left(\boldsymbol{k}, Q_{1}\right)\left(\frac{Q_{1}}{k}\right)^{\omega} \mathcal{G}_{\omega}\left(\boldsymbol{k}, \boldsymbol{k}^{\prime}\right) \Phi_{\gamma^{*}}\left(\boldsymbol{k}^{\prime}, Q_{2}\right)\left(\frac{Q_{2}}{k^{\prime}}\right)^{\omega} .
\end{aligned}
$$

It seems natural to absorb the additional factors $\left(\frac{Q_{1}}{k}\right)^{\omega}$ and $\left(\frac{Q_{2}}{k^{\prime}}\right)^{\omega}$ into the impact factors of the photon. We introduce the Mellin transforms

$$
\begin{aligned}
\Phi_{\gamma^{*}}\left(\boldsymbol{k}, Q^{2}\right) & =\int \frac{d \gamma}{2 \pi i}\left(\frac{Q^{2}}{k^{2}}\right)^{\gamma} \psi(\gamma) \\
\mathcal{G}_{\omega}\left(\boldsymbol{k}, \boldsymbol{k}^{\prime}\right) & =\int \frac{d \gamma}{2 \pi i} \frac{1}{k k^{\prime}}\left(\frac{k^{2}}{k^{\prime 2}}\right)^{\gamma} \frac{1}{\omega-\chi(\gamma)},
\end{aligned}
$$


where $\chi(\gamma)$ is the eigenvalue function of the BFKL kernel (for simplicity we ignore the scale dependence of the strong coupling $\alpha_{s}$ ). Substituting these Mellin transforms in the cross section formula (84) and evaluating the integrals over $\boldsymbol{k}$ and $\boldsymbol{k}^{\prime}$ (which lead to $\delta$-functions in the $\gamma^{\prime}$ 's), we obtain the following result for the cross section,

$$
\begin{aligned}
\sigma_{\gamma^{*} \gamma^{*}}(s)= & \frac{1}{4} \frac{1}{\sqrt{Q_{1}^{2} Q_{2}^{2}}} \int_{\delta-i \infty}^{\delta+i \infty} \frac{d \omega}{2 \pi i}\left(\frac{s}{Q_{1} Q_{2}}\right)^{\omega} \\
& \times \int \frac{d \gamma}{2 \pi i}\left(\frac{Q_{1}^{2}}{Q_{2}^{2}}\right)^{\gamma} \frac{\psi\left(\gamma-\frac{\omega}{2}-\frac{1}{2}\right) \psi\left(-\gamma-\frac{\omega}{2}-\frac{1}{2}\right)}{\omega-\chi(\gamma)} .
\end{aligned}
$$

The change of the energy scale therefore leads to shifts in the $\gamma$ variables [12, 13].

An important consistency is the agreement with the renormalization group equation. If we consider the DIS limit $Q_{1}^{2} \gg Q_{2}^{2}$, our result has to agree with the NLO result of the DGLAP scheme, restricted to the neighbourhood of the point $\omega=0$ (here $n=\omega+1$ is the moment index). It is easy to see that in the limit $Q_{1}^{2} \gg Q_{2}^{2}$ our formula can be rewritten in the following way:

$$
\sigma_{\gamma^{*} \gamma^{*}}(s)=\frac{1}{4 Q_{1}^{2}} \int_{\delta-i \infty}^{\delta+i \infty} \frac{d \omega}{2 \pi i}\left(\frac{1}{x_{B}}\right)^{\omega} \int \frac{d \gamma}{2 \pi i}\left(\frac{Q_{1}^{2}}{Q_{2}^{2}}\right)^{\gamma} \frac{\psi(\gamma-\omega-1) \psi(-\gamma)}{\omega-\chi\left(\gamma-\frac{\omega}{2}-\frac{1}{2}\right)} .
$$

This last equation provides a stringent test for the choice of the cutoff parameter of $\alpha_{\ell}$. Previously, in [12,13] the impact factors for on-shell quarks and gluons have been considered: here the cutoff parameter has been derived from the angular ordering condition, together with the requirement that the collinear singularities are reproduced correctly.

\section{THE $q \bar{q} g$-COMPONENT OF THE PHOTON WAVE FUNCTION}

Let us next address the question how our results fit into the picture of the photon wave function and the color dipole cross section. We expect that, if this picture remains valid in NLO, our results should lead to a new Fock component of the photon, the $q \bar{q} g$ component. For a precise definition we first have to define what we mean, in NLO, by $q \bar{q}$ component. As an example, a $q \bar{q} g$ state of the photon where the gluon is either soft or collinear with the quark (or antiquark), should be counted as a part of the $q \bar{q}$ component. These contributions contain the infrared singularities which cancel against those of the virtual corrections. Consequently, the NLO definition of the $q \bar{q}$ component requires a 'resolution scale' which, in the $q \bar{q} g$ state of the photon, separates the $q \bar{q}$ component from the $q \bar{q} g$ component.

As a first step, we consider our results (35)-(64), and ignore both the infrared singularities and the subtraction of the central region discussed in the previous section. We will show that our results can be expressed in terms of a new photon wave function, $\psi_{q \bar{q} g}\left(Q^{2} ; \boldsymbol{\rho}_{1}, \boldsymbol{\rho}_{2}, \alpha, \alpha_{\ell}\right)$, and the interaction cross section $\sigma_{q \bar{g} g}\left(\alpha, \alpha_{l}, \boldsymbol{\rho}_{1}, \boldsymbol{\rho}_{2}\right)$. To this end, we Fourier transform into transverse configuration space. We begin with the terms proportional to $\boldsymbol{A}_{i} \boldsymbol{A}_{j}$, which are present in all $\mathcal{A A}_{i j}$ (except for the elements with $j=5$ ). As an illustration, consider the contribution $\mathcal{A A}_{11}$ to (32). Obviously we can write

$$
\mathcal{A A}_{11}=\frac{\left(\boldsymbol{A}_{1}\right)_{m}}{D_{1} D_{2}}\left(2 \delta_{m n} C_{F} \alpha_{1} \bar{\alpha}_{1}\right) \frac{\left(\boldsymbol{A}_{1}\right)_{n}}{D_{1} D_{2}} .
$$


(where the sum over $m$ and $n$ is included). Defining the Fourier transform of the last factor:

$$
\iint \frac{d^{2} \boldsymbol{k} d^{2} \boldsymbol{\ell}}{(2 \pi)^{2}} e^{i \boldsymbol{\rho}_{1} \boldsymbol{k}} e^{i \boldsymbol{\rho}_{2} \boldsymbol{l}} \frac{\left(\boldsymbol{A}_{1}\right)_{n}}{D_{1} D_{2}}
$$

and using (17), (18), and (35), we note that the dependence of the integrand $\frac{\left(\boldsymbol{A}_{1}\right)_{n}}{D_{1} D_{2}}$ upon $\boldsymbol{r}$ is only through the combination $\boldsymbol{k}+\boldsymbol{r}$. We therefore change the integration variable $\boldsymbol{k}$ into $\boldsymbol{k}^{\prime}=\boldsymbol{k}+\boldsymbol{r}$ and obtain

$$
N \sqrt{\frac{Q^{2}}{\alpha_{2} \bar{\alpha}_{1}}} \iint \frac{d^{2} \boldsymbol{k} d^{2} \boldsymbol{\ell}}{(2 \pi)^{2}} e^{i \boldsymbol{\rho}_{1} \boldsymbol{k}} e^{i \boldsymbol{\rho}_{2} \boldsymbol{l}} \frac{\left(\boldsymbol{A}_{1}\right)_{n}}{D_{1} D_{2}}=e^{-i \boldsymbol{\rho}_{1} \boldsymbol{r}} \psi_{q \bar{q} ; n}^{(1)}\left(Q^{2} ; \boldsymbol{\rho}_{1}, \boldsymbol{\rho}_{2}, \alpha, \alpha_{\ell}\right),
$$

where

$$
\psi_{q \bar{q} g ; n}^{(1)}\left(Q^{2} ; \boldsymbol{\rho}_{1}, \boldsymbol{\rho}_{2}, \alpha, \alpha_{\ell}\right)=N \sqrt{\frac{Q^{2}}{\alpha_{2} \bar{\alpha}_{1}}} \iint \frac{d^{2} \boldsymbol{k}^{\prime} d^{2} \boldsymbol{\ell}}{(2 \pi)^{2}} e^{i \boldsymbol{\rho}_{1} \boldsymbol{k}^{\prime}} e^{i \boldsymbol{\rho}_{2} \ell} \frac{\left(\boldsymbol{A}_{1}\right)_{n}}{D_{1} D_{2}}
$$

has no dependence upon $\boldsymbol{r}$. Eq. (92) therefore defines a new Fock component of the (longitudinal) photon wave function. $N$ is the normalization constant which can be determined only in combination with the NLO $q \bar{q}$ Fock-component. With a similar result for the first term in (89), $\mathcal{A \mathcal { A }}_{11}$ takes the form:

$$
\begin{aligned}
\frac{Q^{2} N^{2}}{\alpha_{2} \bar{\alpha}_{1}} \mathcal{A A}_{11}= & \int \frac{d^{2} \boldsymbol{\rho}_{1}^{\prime} d^{2} \boldsymbol{\rho}_{2}^{\prime}}{\left(2 \pi^{2}\right)} e^{i \boldsymbol{\rho}_{1}^{\prime} \boldsymbol{k}} e^{i \boldsymbol{\rho}_{2}^{\prime} \boldsymbol{l}} e^{i \boldsymbol{\rho}_{1}^{\prime} \boldsymbol{r}} \psi_{q \bar{q} g ; n}^{(1)}\left(Q^{2} ; \boldsymbol{\rho}_{1}^{\prime}, \boldsymbol{\rho}_{2}^{\prime}, \alpha, \alpha_{\ell}\right)^{*}\left(2 \delta_{m n} C_{F} \alpha_{1} \bar{\alpha}_{1}\right) \\
& \times \int \frac{d^{2} \boldsymbol{\rho}_{1} d^{2} \boldsymbol{\rho}_{2}}{\left(2 \pi^{2}\right)} e^{-i \boldsymbol{\rho}_{1} \boldsymbol{k}} e^{-i \boldsymbol{\rho}_{2} \boldsymbol{l}} e^{-i \boldsymbol{\rho}_{1} \boldsymbol{r}} \psi_{q \bar{q} g ; n}^{(1)}\left(Q^{2} ; \boldsymbol{\rho}_{1}, \boldsymbol{\rho}_{2}, \alpha, \alpha_{\ell}\right) .
\end{aligned}
$$

Integrating over the transverse momenta of the $q \bar{q} g$ system, we obtain $\delta$-functions in the $\boldsymbol{\rho}$-vectors which allow to do the $\boldsymbol{\rho}_{1}^{\prime}, \boldsymbol{\rho}_{2}^{\prime}$ integrals:

$$
Q^{2} N^{2} \int \frac{d \alpha d \alpha_{\ell} d^{2} \boldsymbol{k} d^{2} \boldsymbol{\ell} d^{2} \boldsymbol{r}}{\alpha_{1} \bar{\alpha}_{1} \alpha_{\ell}} \mathcal{A A}_{11}=\int d \alpha d \alpha_{\ell} \int d^{2} \boldsymbol{\rho}_{1} d^{2} \boldsymbol{\rho}_{2}\left(\psi_{q \bar{q} g ; m}^{(1)}\right)^{*} \sigma_{q \bar{q} g ; m n}^{(11)} \psi_{q \bar{q} g ; n}^{(1)},
$$

where

$$
\sigma_{q \bar{q} g ; m n}^{(11)}\left(\alpha, \alpha_{l}, \boldsymbol{\rho}_{1}, \boldsymbol{\rho}_{2}\right)=\frac{1}{\alpha_{\ell}} \int d^{2} \boldsymbol{r} e^{i \boldsymbol{\rho}_{1} \boldsymbol{r}}\left(2 \delta_{m n} \frac{C_{F} \alpha_{1} \bar{\alpha}_{1}}{\boldsymbol{r}^{4}}\right) e^{-i \boldsymbol{\rho}_{1} \boldsymbol{r}} .
$$

The final definition of $\sigma_{q \bar{q} g ; m n}^{(11)}\left(\alpha, \alpha_{l}, \boldsymbol{\rho}_{1}, \boldsymbol{\rho}_{2}\right)$ requires an overall constant which cannot be determined at this stage. The incoming and the outgoing photons have the same transverse coordinates, i.e. the transverse coordinates of the $q \bar{q} g$ system remain 'frozen' during the interaction with the lower quark. We consider this as an important feature of the real corrections. 
It is easy to see that a similar analysis applies to the terms proportional to $\boldsymbol{A}_{i} \boldsymbol{A}_{j}$ in all other $\mathcal{A A}_{i j}$. In summary, we transform the transverse momenta in the following way:

$$
\begin{array}{lll}
\frac{\boldsymbol{A}_{1}}{D_{1} D_{2}}: & \boldsymbol{k}^{\prime}=\boldsymbol{k}+\boldsymbol{r} & \boldsymbol{\ell}^{\prime}=\boldsymbol{\ell} \\
\frac{\boldsymbol{A}_{2}}{D_{2} D_{4}}: & \boldsymbol{k}^{\prime}=\boldsymbol{k}+\boldsymbol{r} & \boldsymbol{\ell}^{\prime}=\boldsymbol{\ell}-\frac{\alpha_{\ell}}{1-\alpha} \boldsymbol{r} \\
\frac{\boldsymbol{A}_{3}}{D_{1} D_{3}}: & \boldsymbol{k}^{\prime}=\boldsymbol{k}+\boldsymbol{r} & \boldsymbol{\ell}^{\prime}=\boldsymbol{\ell} \\
\frac{\boldsymbol{A}_{4}}{D_{2} D_{5}}: & \boldsymbol{k}^{\prime}=\boldsymbol{k}+\boldsymbol{r} & \boldsymbol{\ell}^{\prime}=\boldsymbol{\ell}-\boldsymbol{r} .
\end{array}
$$

In this way we eliminate the dependence upon $\boldsymbol{r}$ from the Fourier transforms and obtain expressions which, after insertion into (32), can be interpreted as photon wave function components $\psi_{q \bar{q} g}^{(i)}$. The $\boldsymbol{r}$ dependence is entirely carried by the interaction cross sections $\sigma_{q \bar{q} g}^{i j}$, similar as in the $q \bar{q}$ case. We carry out the transformation of the elements $\boldsymbol{B}_{i}$ in a similar fashion. The shifts in the $\boldsymbol{k}$ and $\boldsymbol{\ell}$ integrations are just transformed according to (11), (12).

As the next class of terms, consider the terms proportional to $\boldsymbol{r}^{2}$. Starting with $\mathcal{A A}_{12}$, we proceed as before, but the Fourier transformation is done only for $\frac{1}{D_{1} D_{2}}$ and $\frac{1}{D_{2} D_{4}}$, respectively: using the same shift of momenta as before, we again find the dependence upon $\boldsymbol{r}$ concentrated in phase factors, and the remaining integrals define new (scalar) contributions to the photon wave functions $\psi_{q \bar{q} g}^{(1)}$ and $\psi_{q \bar{q} g}^{(2)}$ which do not depend upon $\boldsymbol{r}$. The factor $\boldsymbol{r}^{2}$ goes into the cross section $\sigma_{q \bar{q} g}^{(12)}$.

Next those contributions to $\mathcal{A A}_{i j}$ which contain $D_{2}$ or $D_{7}$ in the numerator: the first appearance is in $\mathcal{A A}_{14}$, and a quick survey shows that these terms show up only if $i=4$ or $j=4$. Therefore, these pieces have to be part of the wave function component $\psi_{q \bar{q} g}^{(4)}$ : e.g. $\frac{D_{2}}{D_{2} D_{5}}$ or $\frac{D_{7}}{D_{2} D_{5}}$. Again, the dependence upon $\boldsymbol{r}$ can be factored out and leads to a phase factor which becomes part of the interaction cross section.

Finally, we have contributions proportional to $\boldsymbol{A}_{i} \boldsymbol{r}$ which appear only for $j=4$ : following again the same steps as before, we take the vector $\boldsymbol{A}_{i}$ as a part of the wave function $\psi_{q \bar{q} g}^{(i)}$, and we absorb $\boldsymbol{r}$ into the cross section $\sigma^{(i 4)}$. The contributions proportional to scalar products such as $(\boldsymbol{k}+\boldsymbol{r})(\boldsymbol{k}+\boldsymbol{\ell})$ are treated in a similar way: one of the two vectors belongs to the wave function $\left(\psi^{(i)}\right)^{*}$, the other into $\psi^{(j)}$ (there is some freedom of how this division is done). In any case, the dependence upon $\boldsymbol{r}$ can entirely be absorbed into the interaction cross sections $\sigma_{q \bar{q} g}$.

As result, all terms appearing on the rhs of $\mathcal{A A}_{i j}, \mathcal{A B}_{i j}$ etc. can be cast either into the form (94), or

$$
\int d \alpha d \alpha_{\ell} \int d^{2} \boldsymbol{\rho}_{1} d^{2} \boldsymbol{\rho}_{2}\left(\psi_{q \bar{q} g: m}^{(i)}\right)^{*} \sigma_{q \bar{q} g ; m}^{(i j)} \psi_{q \bar{q} g}^{(j)}
$$

or

$$
\int d \alpha d \alpha_{\ell} \int d^{2} \boldsymbol{\rho}_{1} d^{2} \boldsymbol{\rho}_{2}\left(\psi_{q \bar{q} g}^{(i)}\right) \sigma_{q \bar{q} g}^{(i j)} \psi_{q \bar{q} g}^{(j)}
$$


In other words, we have both scalar and vector-like contributions to the new photon wave function $\psi_{q \bar{q} g}$.

At this stage, the matrix of interaction cross sections $\sigma^{(i j)}$ (here the indices $i$ and $j$ have to be extended in order to include $\mathcal{A} \mathcal{A}, \mathcal{A B}, \mathcal{B} \mathcal{A}$, and $\mathcal{B B}$, i.e. they run from 1 to 10 ) is not diagonal: so far our choice of the basis of the wave function components had been dictated by the Feynman diagrams from which we have started our calculations. Since the matrix is symmetric, we can always find a more physical basis in which the interactions are diagonal. In this basis, our cross section can be written as a sum of squares, each of which supports the wave function interpretation. In particular, the transverse distances in the incoming and outgoing wave functions are the same: they remain 'frozen' during the interaction with the quark target. Note that the definition of eigenstates of the $q \bar{q} g$-system is determined by the interaction cross section: it is the interaction with the quark which filters out the eigenstates of the $q \bar{q} g$-system.

As we have indicated before, this analysis is not yet complete. To proceed further, we have to combine the results of this paper with the virtual corrections obtained in [1]: a suitable definition of the NLO $q \bar{q}$ state requires a subtraction of a part of the phase space of the $q \bar{q} g$ final state. Together with the subtraction of the central region, this may very well lead to a 'soft melting' of the 'frozen' transverse distances. This would not be totally unexpected: a soft gluon occupies a large region in transverse space, and it may take the form of a non-local cloud around the quark or antiquark.

\section{CONCLUSIONS}

In this paper we have computed, for a longitudinally polarized virtual photon, the helicity-summed squared matrix element of the process $\gamma^{*}+q \rightarrow(q \bar{q} g)+q$. The calculation is valid in the Regge limit with a large rapidity gap between the $q \bar{q} g$-system and the other outgoing quark. The results are of interest for several different lines of research. In each case, further calculations are needed which will be based upon the results of this paper.

First of all, our results present the real corrections to the NLO corrections of the photon impact factor. In order to complete the NLO calculations we have to integrate over the final momenta of the $q \bar{q} g$-system and to combine with the virtual corrections obtained in [1]. In the sum of the real and virtual corrections the infrared singularities have to cancel; furthermore, we have to subtract the contribution of the central region.

Next, the results of this paper contain the three-parton final state of the photon-gluon fusion process $\gamma^{*}+g \rightarrow q \bar{q} g$ where the incoming gluon is off-shell. The complete NLO calculation of this process also requires, in addition to the tree-level calculation of the process $\gamma^{*}+g \rightarrow q \bar{q} g$, to consider the sum with the virtual corrections to $\gamma^{*}+g \rightarrow q \bar{q}$ (which is contained in [1]). From the $q \bar{q} g$ final states we have to subtract the central region. With a suitable jet definition, one obtains the two-jet and the three-jet cross sections in the $k_{T^{-}}$ factorization scheme.

Finally, our results are part of the NLO corrections to the photon wave function picture. Ignoring infrared singularities and the problem of the central region, we have calculated the new $q \bar{q} g$ Fock component of the photon wave function. However, for a proper treatment of the infrared part we need to consider also the virtual corrections in [1]: we have to define a 
NLO $q \bar{q}$ Fock state which includes a part of the soft gluons in our $q \bar{q} g$ final state.

Note added: Recently, a paper by V. S. Fadin, D. Y. Ivanov and M. I. Kotsky has appeared which also addresses the NLO calculation of the virtual photon impact factor (hep-

ph/0106099). It summarizes the status of the virtual corrections, repeating the content of hep-ph/0007119, and it presents the matrix elements for the process $\gamma^{*}+q \rightarrow(q \bar{q} g)+q$. In our paper we have calculated the helicity sum of the squared matrix elements for this process.

\section{ACKNOWLEDGMENTS}

Very helpful discussions with D. Colferai, J. Collins and C.-F. Qiao are gratefully acknowledged. One of us (J.B.) has profited from a useful discussion with M. Ciafaloni.

[1] J. Bartels, S. Gieseke and C. F. Qiao, Phys. Rev. D 63 (2001) 056014 hep-ph/0009102].

[2] S.Catani, M.Ciafaloni, and F.Hautman, Phys. Lett. B242 (1990) 97; Nucl. Phys. B366 (1991) 135.

[3] A. H. Mueller, Nucl. Phys. B 335 (1990) 115.

[4] N. N. Nikolaev and B. G. Zakharov, Z. Phys. C 49 (1991) 607.

[5] K. Golec-Biernat and M. Wüsthoff, Phys. Rev. D 59 (1999) 014017 [hep-ph/9807513].

[6] K. Golec-Biernat and M. Wüsthoff, Phys. Rev. D 60 (1999) 114023 [hep-ph/9903358].

[7] K. Golec-Biernat and M. Wusthoff, Eur. Phys. J. C 20 (2001) 313 hep-ph/0102093.

[8] V. S. Fadin, R. Fiore, M. I. Kotsky and A. Papa, Phys. Rev. D 61 (2000) 094006 [hep$\mathrm{ph} / 9908265$.

[9] V. S. Fadin and A. D. Martin, Phys. Rev. D 60 (1999) 114008 hep-ph/9904505.

[10] R. Mertig, M. Böhm and A. Denner, Comput. Phys. Commun. 64 (1991) 345.

[11] E. A. Kuraev, L. N. Lipatov and V. S. Fadin, Sov. Phys. JETP 45 (1977) 199 [Zh. Eksp. Teor. Fiz. 72 (1977) 377]; I. I. Balitsky and L. N. Lipatov, Sov. J. Nucl. Phys. 28 (1978) 822 [Yad. Fiz. 28 (1978) 1597].

[12] M. Ciafaloni, D. Colferai and G. P. Salam, Phys. Rev. D 60 (1999) 114036 [hep-ph/9905566].

[13] D. Colferai, PhD Thesis, University of Florence 1999, hep-ph/0008309. 